\title{
On the Effect of the Fiber Orientation on the Flexural Stiffness of Injection Molded Short Fiber Reinforced Polycarbonate Plates
}

\author{
N. M. NEVES, G. ISDELL, and A. S. POUZADA \\ Department of Polymer Engineering, Universidade do Minho \\ Campus de Azurém, 4800 Guimarães, Portugal \\ and \\ P. C. POWELL \\ Chair of Eng. Design with Plastics, Dept. of Mech. Eng. \\ University of Twente \\ 7500 AE Enschede, The Netherlands
}

\begin{abstract}
The through-thickness fiber orientation distribution of injection molded polycarbonate plates was experimentally determined by light reflection microscopy and manual digitization of polished cross sections. Fiber length distribution was determined by pyrolysis tests followed by image analysis. A statistical analysis was done to determine the confidence limits of the fiber orientation results. The fiber orientation distribution was described by using second-order orientation tensors. The throughthickness stiffness variations were determined by the orientation averaging approach. This layer stiffness distribution was used to simulate the behavior of beams subjected to three point bending with a FEM Ansys model. The results were compared with experimentally determined flexural stiffness both in the flow direction and in the transverse flow direction. The effect of flow rate and melt-temperature on stiffness and fiber orientation is discussed.
\end{abstract}

\section{INTRODUCTION}

$I^{n}$ njection molded short fiber reinforced thermoplastic composites are increasingly used in engineering parts since the properties of the base material can be improved or tailored through the reinforcement. This is achieved without significant loss of productivity and reproducibility of the injection molding process. Of course, some compromise is needed since the surface finish of these products is poor, and the fiber length and volume fraction are limited in order to keep the material with viscosity suitable for injection molding. The analysis and modeling of the mechanical behavior of these materials involve either the determination or the prediction of the state of fiber orientation after molding. Modeling and prediction of the fiber orientation resulting from the flow of discontinuous fiber reinforced thermoplastics is a relevant theme of research (1-13). Until now, some aspects have not been fully developed, such as the fiber interaction during flow or the determination of the fiber orientation distribution in fiber reinforced products.
In order to determine the state of orientation of the fibers it is necessary to obtain statistically representative specimens from representative regions of the products. The fiber orientation in those specimens can be studied by light reflection microscopy (14-17). The sections of the fibers will appear as circles, ellipses, or rectangles, according to the direction of penetration in the matrix. By digitization of the axis endpoints of the circles and ellipses, and length and width of the rectangles, it is possible to calculate the distribution of fiber orientation in terms of the in-sectioning-plane angle, $\phi$, and out-of-sectioning-plane angle, $\theta$. However, the analysis of sections does not enable the determination of the complete three-dimensional state of fiber orientation. In fact, from the geometry of the ellipse, it is not possible to distinguish between the two possible directions of penetration in the matrix. There are other problems involved, such as the fact that one section is always a biased sample. The longer fibers and the fibers whose axial directions of alignment are perpendicular to the section have greater probability 
of appearing in the cut section. Some corrections derived from stereology are applicable in order to minimize the errors $(14,18)$.

To overcome the ambiguity of the measurements, other techniques have been suggested, the most promising being the optical sectioning method using confocal laser scanning microscopy (19-21). The idea behind this method is: if the focus point of a laser beam can be moved in depth inside the polished section, it is then possible to trace the direction of penetration of the fiber in the matrix. The technique can be automated so that a mechanical device is able to control both the depth of focus of the laser and the in-plane movement of the specimen mounted in the microscope. The only drawback of the technique, as pointed out by Clark (19), is that some limitations exist resulting from the material transparency and the percentage of fiber. Other authors have suggested and used other techniques, which are hereby referred to as a matter of record: contact microradiography (22); scanning electron microscopy (23); ultrasonic velocity measuring (24), and Frauenhofer diffraction of photograph negatives from polished sections $(25,26)$.

The fiber orientation data can be described in the form of a probability distribution function, $\psi(\theta, \phi)$, orientation factors (27) or orientation tensors (6). All of them having their own merits, the choice is made according with the data handling capacity and the end use of the data. When the objective is to predict the mechanical properties, the orientation factors and the orientation tensors are preferred. Further, the orientation tensors can completely characterize the fiber orientation and be directly used in calculating properties.

From the fiber orientation state in a product, it is possible to predict mechanical properties such as stiffness, compliance, or linear thermal expansion coefficients. The method requires knowledge of the properties of a uniaxial ply, which in most cases is not readily available. The mechanical properties of a ply can be estimated from the matrix and fiber properties, using the empirical Halpin-Tsai equation (28). Combining the uniaxial ply properties with the distribution of fiber orientation, the so-called orientation averaged properties can be obtained (6). The concept is analogous to that used for laminates, which consists in averaging the contribution of the two phases to determine the properties of an equivalent homogeneous material (27). Of course, if one of the phases has orientation dependent properties, the information about the distribution of orientation must be taken into account. However, the approach used in the lamination theory is valid only for continuous fibers with planar or quasiplanar orientation (29). In the case of non-planar orientation of discontinuous short fibers, the laminate theory is not applicable. Injection molded products usually have some out-of-plane orientation, which is usually neglected (6).

In this work, results of through-thickness fiber orientation measurements in glass fiber reinforced poly- carbonate plates are described using the tensors of fiber orientation. The tensors of fiber orientation were used to predict the through-thickness stiffness of the product. In order to evaluate the predictions, three point bending tests were performed using beams cut from the plates. The results of the bending tests are compared with FEM simulations based on the predicted through-thickness stiffness calculated from the fiber orientation data.

\subsection{Fiber Orientation}

The state of orientation of a single fiber can be characterized from the angles $\theta$ and $\phi$ as defined in Fig. 1. The directions 1, 2, and 3 refer to the flow, width and thickness directions, respectively.

The orientation of the fiber can be described by a probability distribution function $\psi(\theta, \phi)$, defined as

$$
\begin{array}{r}
P\left(\theta_{1} \leq \theta \leq \theta_{1}+\delta \theta, \phi_{1} \leq \phi \leq \phi_{1}+\delta \phi\right) \\
=\psi_{(\theta, \phi)} \sin (\theta) d \theta d \phi
\end{array}
$$

An alternative way to describe the orientation of the fiber is by associating a unit vector $\mathbf{p}$ to the fiber direction, as shown in Fig. 1. Thus the distribution function could be written as a function of $\mathbf{p}, \psi_{(\mathbf{p})}$. The components of the vector $\mathbf{p}$ are computed in terms of $\phi$ and $\theta$ as

$$
\begin{gathered}
p_{1}=\cos \theta \\
p_{2}=\sin \theta \sin \phi \\
p_{3}=\sin \theta \cos \phi
\end{gathered}
$$

The distribution function must satisfy some physical requirements. Since the fiber rotated $180^{\circ}$ about the axis $1,\left(\theta, 180^{\circ}+\phi\right)$ is indistinguishable from the fiber $(\theta, \phi)$, the distribution function should be an even function, i.e., $\psi_{(\mathbf{p})}=\psi_{(-\mathbf{p})}$. Thus, only the even order tensors are non-vanishing.

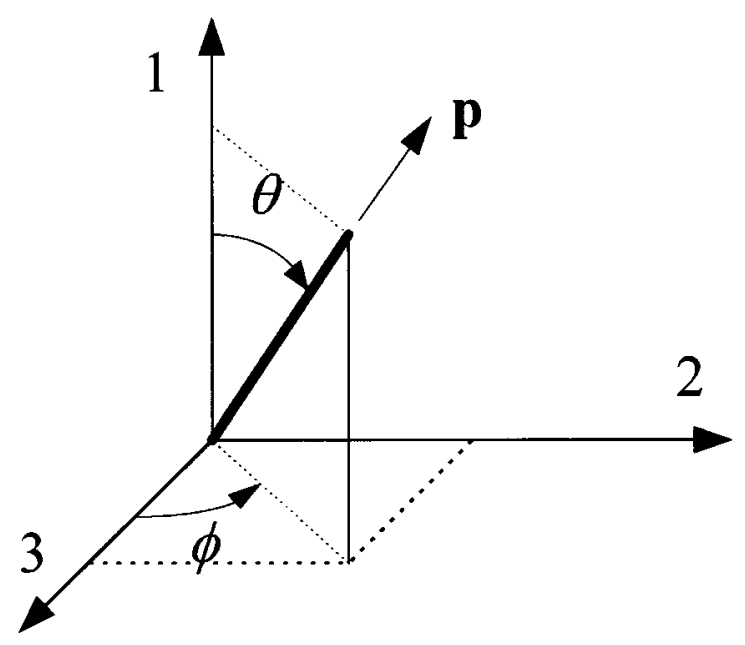

Fig. 1. Definition of the co-ordinate system used, the in plane angle $\phi$, the out of plane angle $\theta$ and the unit vector $\boldsymbol{p}$. The directions 1,2 and 3 refer to flow, transverse flow, and thickness directions, respectively. 
Experimentally, the second- and fourth-order tensors can be calculated from the vector components of p, as:

$$
\begin{gathered}
<a_{i j}>=\frac{\sum_{n}\left(p_{i}\right)_{n}\left(p_{j}\right)_{n} F_{n}}{\sum_{n} F_{n}} \\
<a_{i j k l}>=\frac{\sum_{n}\left(p_{i}\right)_{n}\left(p_{j}\right)_{n}\left(p_{k}\right)_{n}\left(p_{l}\right)_{n} F_{n}}{\sum_{n} F_{n}}
\end{gathered}
$$

where the angle brackets denote the average over $n$ fibers, and $F_{n}=\left(1 / \cos \theta_{n}\right)$ is a bias correction or weighting function. The use of the second- or fourth-order tensor to describe the fiber orientation state depends on the type of distribution obtained from the measurements. Moderately aligned orientation states can be modeled by second-order tensors, while highly aligned states require fourth-order tensors (30). Moreover, higher-order tensors provide complete information about lower-order tensors $\left(a_{i j k k}=a_{i j}\right)$. From the orientation tensors it is also possible to recover the orientation distribution function (6).

The physical meaning of the terms of the secondorder tensor of fiber orientation, a $3 \times 3$ square matrix, can be readily understood. The sum of the diagonal elements must be the unit, since it equals the length of the unit vector $\mathbf{p}$. The value of each element of the diagonal stands for the relative orientation of the fibers around the axis (e.g. $a_{11}=1$ means that all the fibers are aligned in 1-direction, $a_{11}=a_{22}=a_{33}=$ $1 / 3$ stands for random in space orientation). The nondiagonal elements are symmetric and give information about the principal directions and may not coincide with the co-ordinate system used (e.g., in a perfectly orthotropic material, the non-diagonal elements are zero; a value of 0.2 in $a_{12}$ component means that the principal directions of orientation are slightly tilted away from axes 1 and 2).

It is also possible to compute mechanical properties, such as the stiffness, using the fourth-order tensor of fiber orientation $(6,31)$. The fourth-order tensor is needed in that case, since the stiffness tensor is also a fourth-order tensor.

\subsection{Mechanical Properties}

The distribution of fiber orientation can be used to estimate the stiffness of the composite using the concept of orientation average (6) or equivalent homogeneity (27). This concept uses the average of a small volume element to generate average response characteristics that can be associated with an equivalent continuum with homogeneous properties. The volume element is assumed to be large enough to contain many fibers, but still small enough relative to the domain of application of stress. Calculating the properties of the equivalent continuum involves averaging the properties of the two phases, the volume fraction of each phase, and the fiber orientation.

Most micromechanical theories use the properties of a fully aligned and transversely isotropic composite to estimate the properties of a composite with any state of orientation. The calculation involves the determination of a set of invariants of the compliance tensor that is composed with the second- and fourth-order tensors of fiber orientation in order to get the stiffness predictions.

The mechanical properties of unidirectional laminates are dependent on the volume fraction, the size and shape of the fibers and can be described by the Halpin-Tsai equations:

$$
\frac{p}{p_{m}}=\frac{1-\xi \eta V_{f}}{1-\eta V_{f}}
$$

where $p$ corresponds to one of the tensile moduli $\left(E_{11}\right.$, $\left.E_{22}\right)$ or shear moduli $\left(G_{12}, G_{23}\right)$, the subscript 1 representing the fiber direction. The subscripts $f$ and $m$ denote fiber and matrix, respectively, and $\xi$ depends upon the shape of the fibers and packing geometry. The volume fraction of fibers is $V_{f}$ and the parameter $\eta$ is calculated using the following expression

$$
\eta=\frac{p_{f} / p_{m}-1}{p_{f} / p_{m}+\xi}
$$

For discontinuous, oriented fiber composites (28),

$$
\xi E_{11}=2 \frac{L}{d} ; \xi E_{22}=2 ; \xi G_{12}=1 ; \xi G_{23}=\frac{K_{m}}{K_{m}+2 G_{m}}
$$

where $L$ and $d$ are the fiber length and diameter, and $K_{m}$ and $G_{m}$, the bulk and shear modulus of the matrix.

The rule of mixtures was used to obtain the major Poisson ratio,

$$
v_{12}=v_{f} V_{f}+v_{m}\left(1-V_{f}\right)
$$

Assuming that injection molded composites are transversely isotropic, the orientation averaged stiffness has the following form:

$$
\begin{aligned}
<C_{i j k l}>=B_{1}<a_{i j k l}>+ & B_{2}\left(<a_{i j}>\delta_{k l}\right)+ \\
B_{3}\left(<a_{i k}>\delta_{j l}+<\right. & \left.a_{i l}>\delta_{j k}+<a_{j l}>\delta_{i k}+<a_{j k}>\delta_{i l}\right)+ \\
B_{4}\left(\delta_{i j} \delta_{k l}\right) & +B_{5}\left(\delta_{i k} \delta_{j l}+\delta_{i l} \delta_{j k}\right)
\end{aligned}
$$

where the values of $B_{1}$ to $B_{5}$ are invariants of the compliance tensor, which can be determined from the stiffness of the unidirectional composite (6), $\left\langle a_{i j k l}\right\rangle$ and $\left\langle a_{i j}\right\rangle$ are the experimentally determined fourthand the second-order orientation tensors, respectively, and $\delta_{i j}$ are the Kronecker delta components.

In a three point bending test, the through-thickness stresses increase from the midplane to the skin. In this way it is possible to study the differences in stiffness originated from differences in fiber orientation, especially in the specimen skin.

The stiffness prediction in each layer enables the simulation of the bending tests either by the classical 


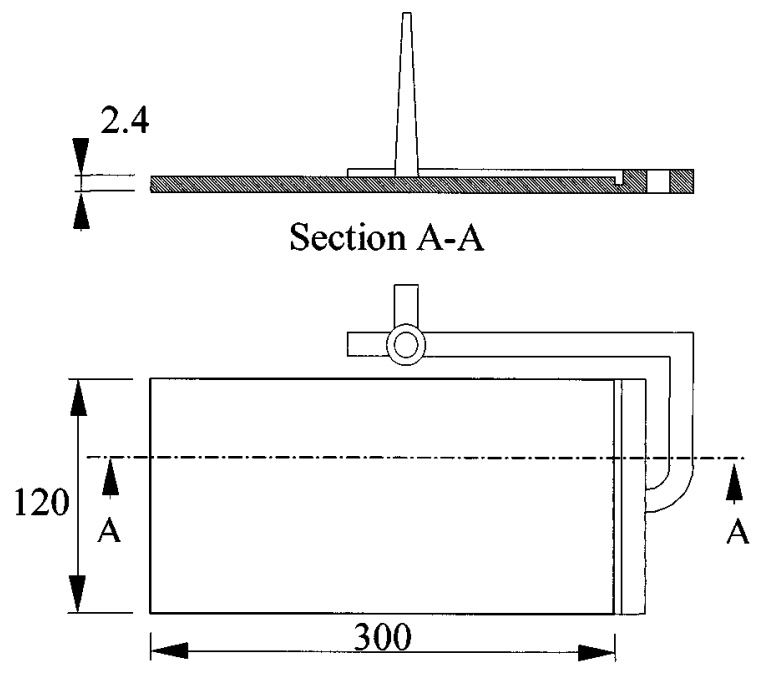

Fig. 2. Geometry of the moldings.

lamination theory or by FEM analysis. In this work, the simulations were made with a FEM model created in Ansys, with four-node square composite elements with 16 symmetrical layers, in plane stress mode. The experimental and simulation results are discussed in terms of the apparent flexural modulus of the material calculated from the following expression:

$$
E_{a p p}=\frac{F S^{3}}{4 y b t^{3}}
$$

where $F$ is the load, $S$ is the span length, $y$ is the deflection in the middle point, $b$ is the width of the beam, and $t$ is the thickness of the beam.

\section{EXPERIMENTAL}

A $10 \%$ by weight glass fiber reinforced polycarbonate grade from General Electric Plastics, Lexan 500 R, was used. According to the manufacturer, the fiber and matrix moduli are 72 and $2.35 \mathrm{GPa}$, respectively, and the respective Poisson ratios are 0.20 and 0.36 . Fiber length and diameter were reported to be about 200 and $10 \mu \mathrm{m}$. The tool used for producing the 300 $\times 120 \times 2.4 \mathrm{~mm}^{3}$ plates is a single impression film gated injection mold. The molding geometry is shown in Fig. 2.
The moldings were obtained with an Engel 175-ton injection-molding machine.

Two melt temperatures, $300^{\circ} \mathrm{C}$ and $320^{\circ} \mathrm{C}$ and three screw forward speeds of 25,40 , and $55 \mathrm{~mm} / \mathrm{s}$ were used. The volume flow rates that correspond to the three screw forward speeds are 49.1, 78.5 and 108.0 $\mathrm{cm}^{3} / \mathrm{s}$, respectively. The mold temperature was kept at $80^{\circ} \mathrm{C}$. The holding pressure was kept constant at $60 \mathrm{MPa}$. The holding time and cooling time were set to $6.5 \mathrm{~s}$ and $20 \mathrm{~s}$, respectively. The corresponding codes are shown in Table 1.

The subscript L or T, when showing, denotes a longitudinal or transverse specimen, respectively. Accordingly, a specimen $300.40 \mathrm{~L}$ means that the specimen was molded at $300^{\circ} \mathrm{C}$, with a $40 \mathrm{~mm} / \mathrm{s}$ screw speed and cut along the flow direction.

From each set of molding conditions, at least 20 plates were molded in stabilized conditions.

\subsection{Fiber Orientation Measurement}

The measurements of fiber orientation were made from polished cross sections cut from the molded plates. Two specimens per molding condition were randomly selected to measure the fiber orientation distribution along and across the flow direction. The specimens used for fiber orientation measurement were cut at $150 \mathrm{~mm}$ from the gate. Figure 3 shows the locations of the specimens and the cutting planes.

The surfaces of samples cut from fiber reinforced parts were subjected to successive stages of polishing (sandpaper of progressively smaller roughness) to achieve a microscopically smooth surface. The sections of the fibers appear in the image as circles if the fibers are aligned in a direction perpendicular to the cut section, rectangles if the fiber axis is parallel to the section, or ellipses otherwise (Fig. 4).

Table 1. Codes for Molding Conditions.

\begin{tabular}{cccc}
\hline & \multicolumn{3}{c}{ Injection Screw Forward Speed } \\
Melt-Temperature $\left({ }^{\circ} \mathbf{C}\right)$ & $\mathbf{2 5}$ & $\mathbf{4 0} / \mathbf{s})$ & $\mathbf{5 5}$ \\
\hline 300 & 300.25 & 300.40 & 300.55 \\
320 & 320.25 & 320.40 & 320.55 \\
\hline
\end{tabular}

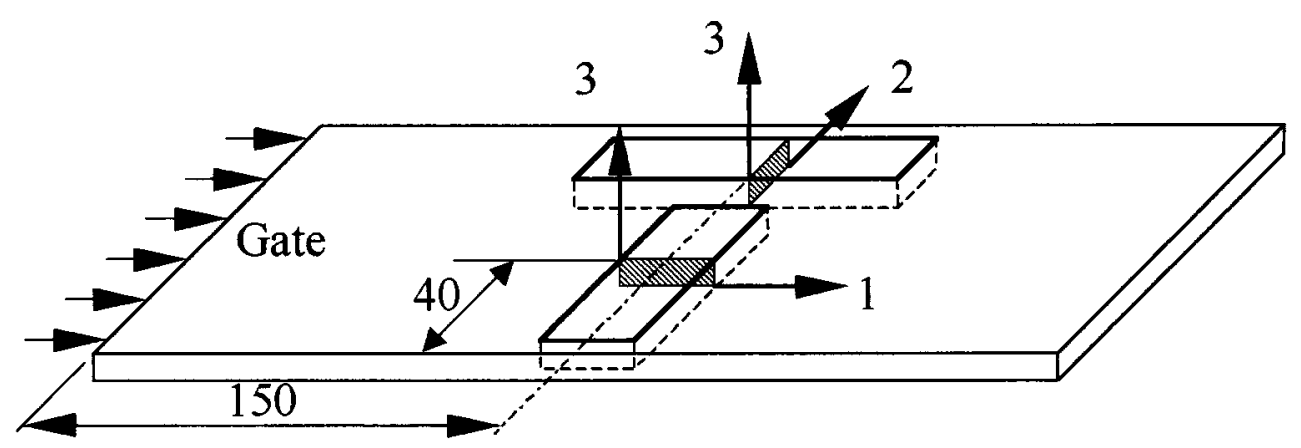

Fig. 3. Specimen for bending tests and fiber orientation measurements. 


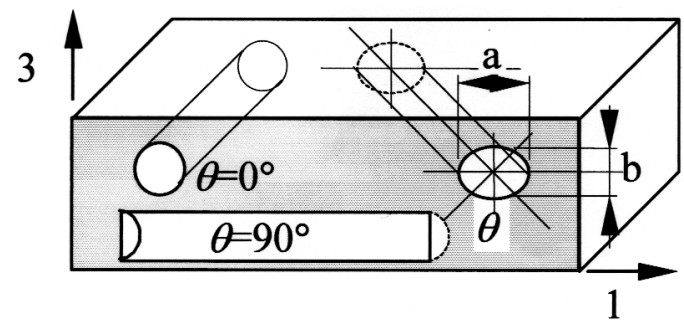

Fig. 4. Possible forms of fiber sections in a polishing surface, $\theta$ is the out-of-plane orientation angle.

The out-of-plane orientation angle $\theta$ is derived from the major and minor axes of the ellipse, $a$ and $b$, as

$$
\theta=\arccos \left(\frac{b}{a}\right)
$$

The results presented here refer to three-dimensional data obtained from one-plane measurements, which mean that the ambiguity in the out-of-diagonal tensor components is present in our results.

The in-plane orientation is determined by the angle defined by the major axis of the ellipse and the preselected reference axes. These angles, $\theta$ and $\phi$, can be determined by digitizing the coordinates of the endpoints of the major and minor axes of the ellipses, either manually or by image analysis.

The relevant components of the unit vector $\mathbf{p}$ for the calculation of the orientation tensor depend on the sectioning plane. If the specimen is cut in the 2-3 plane, the Eqs 2 to 4 can be applied. However, if the specimen is cut in the 1-3 plane, the $p_{1}$ and $p_{2}$ definitions (Eqs 2 and 3) must be switched, in order to obtain the unit vector components, $p_{i}$, in the correct co-ordinate system.

The elements of the second- and fourth-order tensors that describe the fiber orientation can be calculated from the values of the vectors $p_{i}$ using Eqs 5 and 6 , respectively.

The cross section was divided into three columns of sixteen layers with equal thickness (actually the height of the image analyzer screen, at the selected magnification). Of each column only eight layers were analyzed (half cross section). The measurements in various columns enable the evaluation of the sampling error. Both the fourth- and the second-order tensors were inferred from the fiber orientation measurements.

\subsection{Fiber Length Characterization}

To determine the fiber length and diameter, a small specimen cut from the material was subjected to pyrolysis. The specimen was kept at a temperature of $600^{\circ} \mathrm{C}$ during 30 minutes, to volatilize the matrix. The glass fibers were measured using an optical microscope Olympus BH-2 and a Leica Quantimet 500 image analyzer. The lengths and diameters of more than 200 fibers were obtained.

\subsection{Three Point Bending Tests}

Five specimen of each type (transverse and longitudinal) were selected for flexural testing, according to the ASTM D 790 standard, using an Instron 1122 universal tensile testing machine. Specimen nominal length and width were $80 \mathrm{~mm}$ and $15 \mathrm{~mm}$, respectively. The support span, S, was chosen to be $60 \mathrm{~mm}$, in line with the ASTM standard. The crosshead speed in the bending test was set to $2.5 \mathrm{~mm} / \mathrm{min}$.

\subsection{From Fiber Orientation to Properties}

The general tensor equation, which enables the orientation averaging calculation, was shown in Eq 11 . The scalar invariants of the stiffness tensor $B_{1}$ to $B_{5}$ are determined from the known values $C_{i j k l}$ of the stiffness tensor and the correspondent tensors of orientation. For the case of the ply aligned in direction 1, the tensor components $a_{11}$ and $a_{1111}$ are equal to unit and the other components of the second- and fourth-order tensors are zero. Hence, after solving a system of equations, the scalar invariants become:

$$
\begin{gathered}
B_{1}=C_{11}+C_{22}-C_{12}-4 C_{66} \\
B_{2}=C_{12}-C_{23} \\
B_{3}=C_{66}-\frac{1}{2}\left(C_{22}-C_{23}\right) \\
B_{4}=C_{23} \\
B_{5}=\frac{1}{2}\left(C_{22}-C_{23}\right)
\end{gathered}
$$

where $C_{i j}$ are the components of the contracted notation stiffness tensor as defined by Jones (32). The contracted notation compliance tensor for a transversely isotropic material (2-3 symmetry plane) is

$$
S_{i j}=\left[\begin{array}{cccccc}
\frac{1}{E_{1}} & -\frac{v_{21}}{E_{2}} & -\frac{v_{21}}{E_{2}} & 0 & 0 & 0 \\
-\frac{v_{12}}{E_{1}} & \frac{1}{E_{2}} & -\frac{v_{32}}{E 2} & 0 & 0 & 0 \\
-\frac{v_{12}}{E_{1}} & -\frac{v_{23}}{E_{2}} & \frac{1}{E_{2}} & 0 & 0 & 0 \\
0 & 0 & 0 & 2\left(S_{22}-S_{23}\right) & 0 & 0 \\
0 & 0 & 0 & 0 & \frac{1}{G_{12}} & 0 \\
0 & 0 & 0 & 0 & 0 & \frac{1}{G_{12}}
\end{array}\right]
$$

The elements of the contracted notation stiffness tensor can be calculated by inversion of the compliance matrix.

When the invariants are determined, the calculation of the orientation averaged stiffness tensor of each layer is obtained by solving $E q 11$ using the determined second- and fourth-order tensors of fiber orien- 


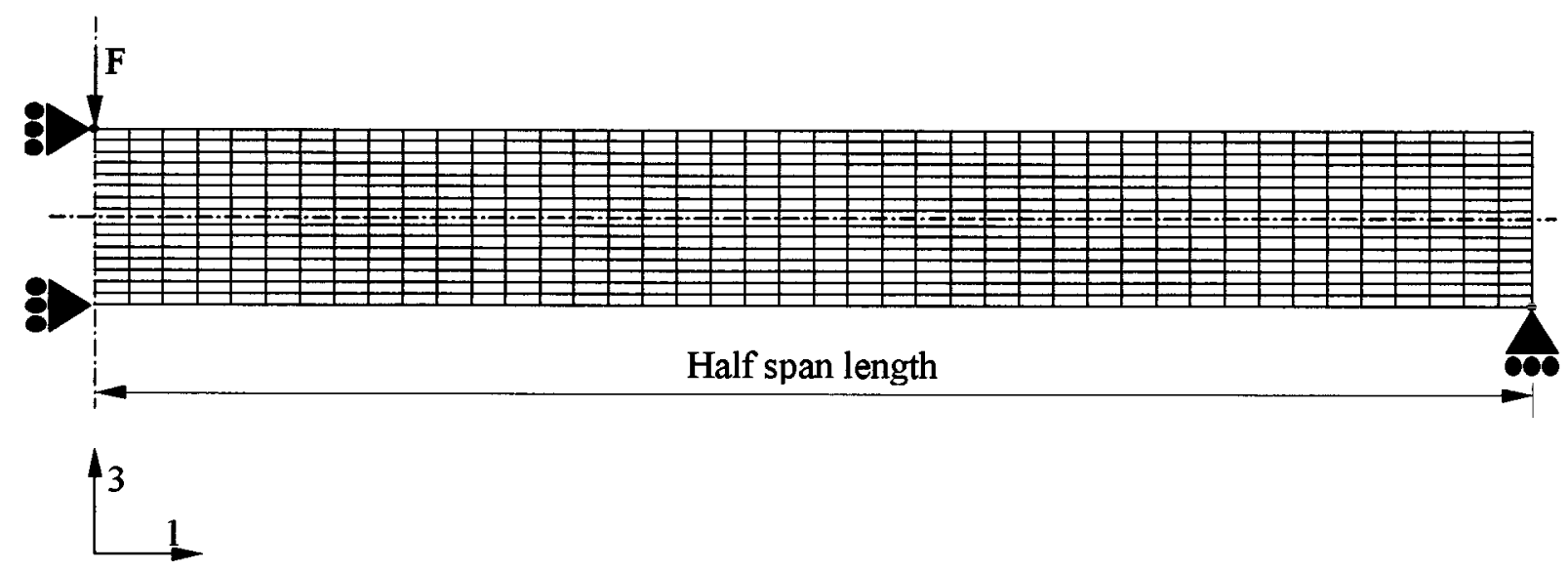

Fig. 5. Ansys model used in simulation of bending tests, showing the boundary conditions.

tation. It is then necessary to transform the stiffness tensor into contracted notation and invert it to obtain the compliance tensor, also in contracted notation. The engineering constants for each layer are readily extracted from the components of the compliance tensor.

\subsection{FEM Model Simulation}

The engineering constants of each layer were introduced in a two-dimensional FEM model created in Ansys. The geometry of the bending test allows the simplification of the model. Because of symmetry only half-span length was modeled. Sixteen symmetric, orthotropic, and uniform-in-thickness layers define the thickness of the beam. The boundary conditions are shown in Fig. 5.

The apparent flexural modulus was calculated from the force and deflection data obtained in the simulations, using $E q 12$.

\section{RESULTS AND DISCUSSION}

\subsection{Fiber Length Results}

The fiber fraction was determined as $10.6 \mathrm{wt} \%\left(V_{f}=\right.$ 5.7). The number average fiber length in the moldings is $L=118 \mu \mathrm{m}$ with a standard deviation of $108 \mu \mathrm{m}$ (Fig. 6). The wide distribution of fiber length is due probably to the fiber breakage during molding. The average fiber diameter is $15 \mu \mathrm{m}$ with a standard deviation of $1 \mu \mathrm{m}$, which is higher than the $10 \mu \mathrm{m}$ manufacturer quoted diameter.

\subsection{Symmetry and Scatter Evaluation}

The distribution of fiber orientation is approximately symmetric about the mid-plane of the part, as was verified for one molding condition and shown in Fig. 7.

Most of the measurements were made only in half of the thickness.

Some correlation exists between the results obtained with specimens cut both in the 1-3 and 2-3 sectioning plane (Figs. 8 and 9). Those results do not match perfectly because of the uncertainty of the measurement of fibers that are perpendicular to the section plane (nearly circles). Similar results were reported by Bay (14).

The overall results indicate the existence of a thin, highly aligned, skin layer $\left(a_{11}\right.$ high). The core is thick and shows a high degree of fiber alignment across the flow direction ( $a_{22}$ high) in all processing conditions.
Fig. 6. Fiber length distribution after processing.

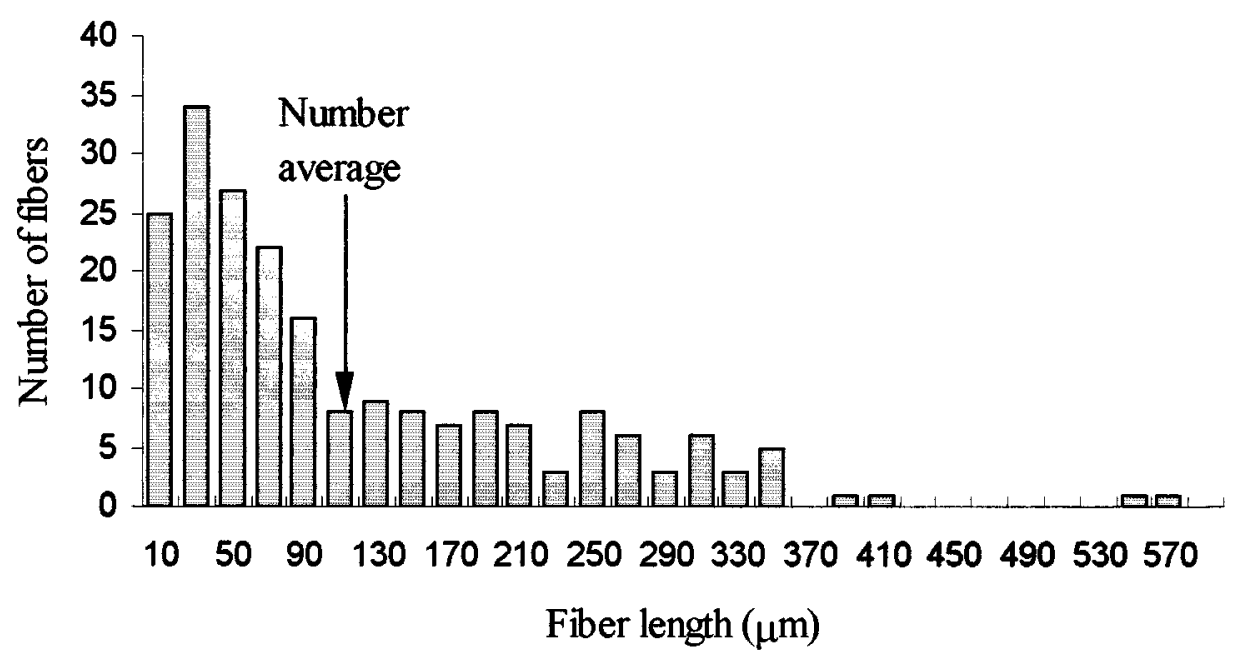




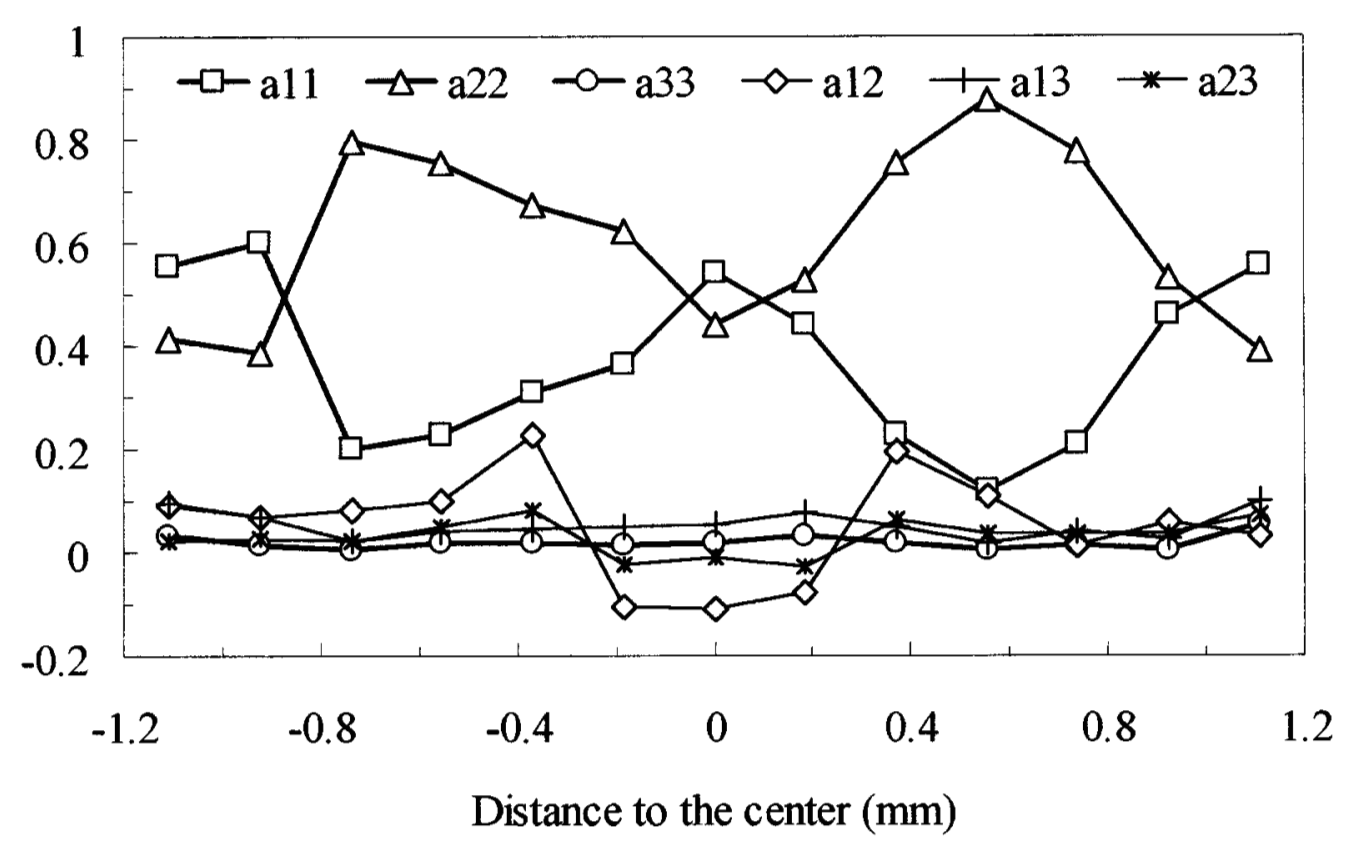

Fig. 7. Tensor components over the complete thickness, specimen 320.55, 2-3 sectioning plane.

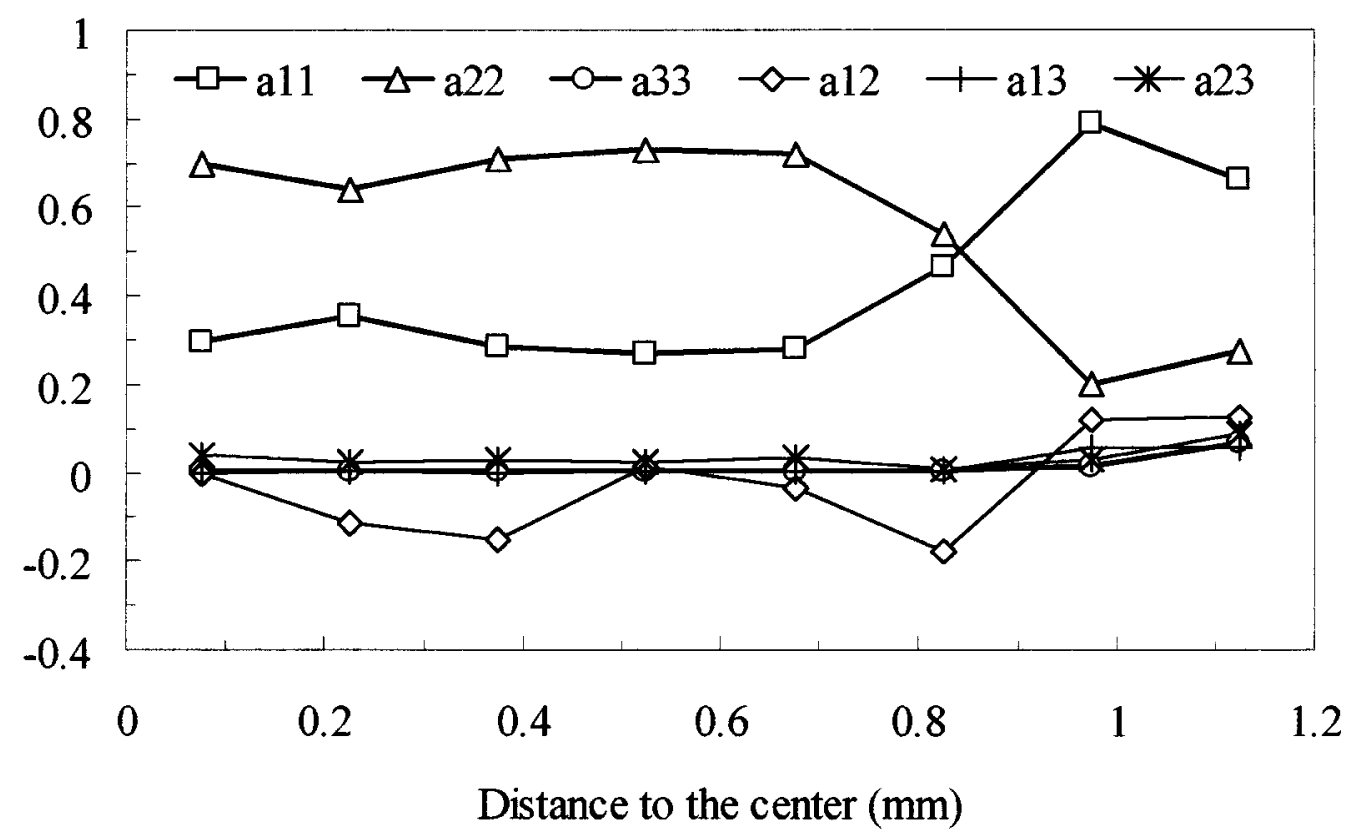

Fig. 8. Sample 300.25, 1-3 sectioning plane.

The values of $a_{33}$ are in most of the cases smaller than 0.01 . This small value indicates that the distribution of fiber orientation is mostly planar. This general result is typical of injection molded parts. Thus, it is possible to discuss the results of fiber orientation only on the basis of $a_{11}$ results since $a_{11}+a_{22} \approx 0.99$.

In some specimens, $a_{12}$ values of the order of 0.3 were observed. This indicates that the directions 1 and 2 are tilted from the principal directions of fiber orientation. However, evidence was found in layer re- moval tests (unpublished) that such misalignment does not exist, because no significant torsion of the beams was observed. This is one of the tensor components that is affected by the ambiguity in the determination of the angle $\theta$. Therefore, it will be assumed that this is an upper bound, the real values being smaller than the computed values.

The measurement errors, associated with the equipment used and experimental procedure, are small (Fig. 10). However, the variance or sampling variation, 


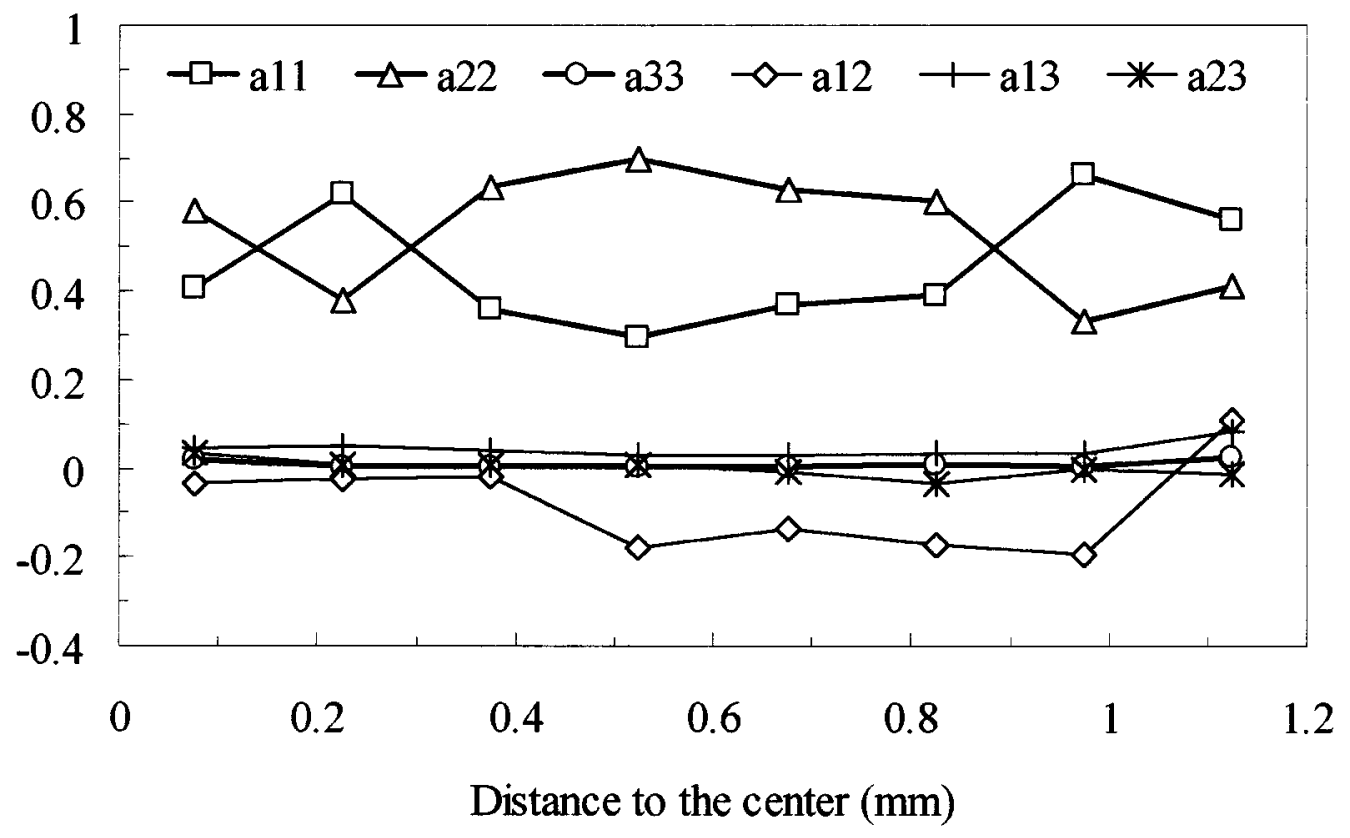

Fig. 9. Sample 300.25, 2-3 sectioning plane.

in Fig. 11, is considerably high. This variation is associated with the number of fibers measured, and the scatter of data from different columns.

The effect of the molding conditions will be discussed on the basis of the $a_{11}$ tensor component obtained from measurements in the 1-3 sectioning plane. The 1-3 sectioning plane measurements give a more accurate description of the fiber orientation at the surface of the molding (longer ellipses). This is why those results are presented instead of those from the 2-3 sectioning plane.

\subsection{Fiber Orientation Results and Discussion}

Slower injection favors the fiber orientation in the flow direction in the outer layers, Fig. 12. From 0.8 to
$1.2 \mathrm{~mm}$ from the center, the fibers are mostly aligned along the flow direction ( $a_{11}$ higher than 0.5).

In the case of the melt temperature at $320^{\circ} \mathrm{C}$ (Fig. 13), the fibers in the outer layers still have a high alignment in the flow direction. The effect of the injection speed is not so apparent at the skin layers, otherwise observed at the inner layers $(0.2$ to $0.7 \mathrm{~mm}$ from the center). A tendency for a more random in-plane orientation is noticeable in the same layers, $\left(a_{11} \approx a_{22}\right.$ $\approx 0.5$ ) if a slower injection speed is used. By increasing it, progressively smaller values of $a_{11}$ will result.

The effect of the molding temperature is more noticeable in the case of slower injection speed. In Fig. 14a it can be observed that the skin is more oriented in the flow direction for lower melt-temperatures, whereas
Fig. 10. Measurement scatter denoted as vertical bars, sample 300.40.

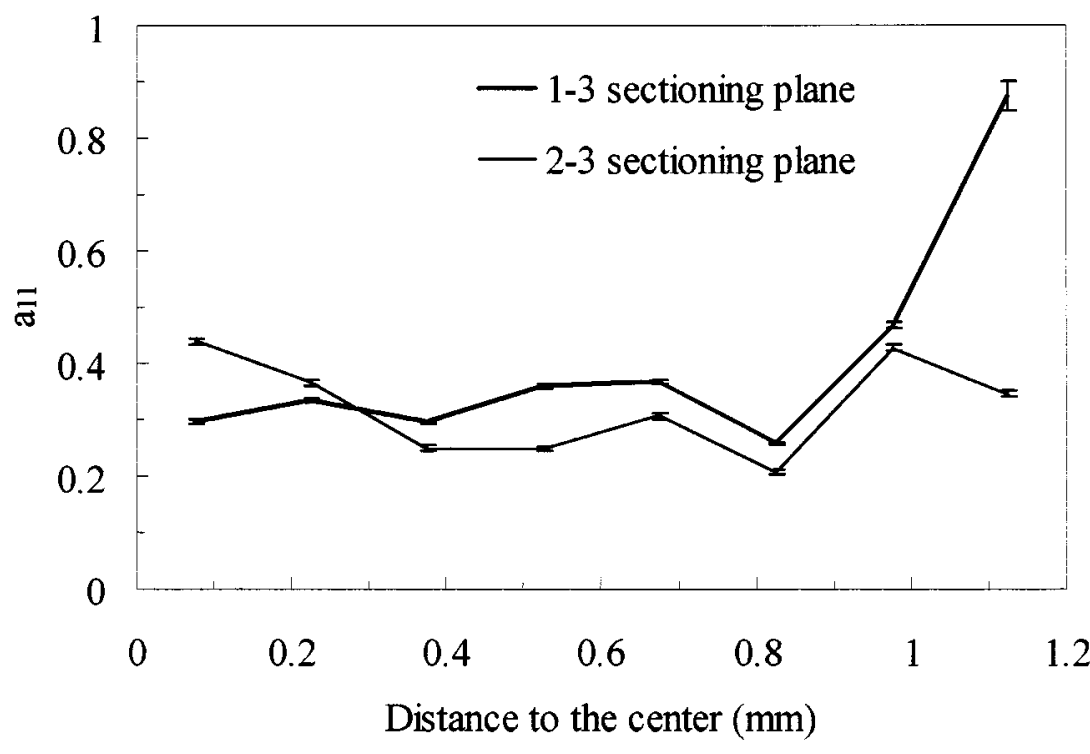


N. M. Neves, G. Isdell, A. S. Pouzada, and P. C. Powell

Fig. 11. Sampling scatter on $a_{11}$, from several specimens 300.40.
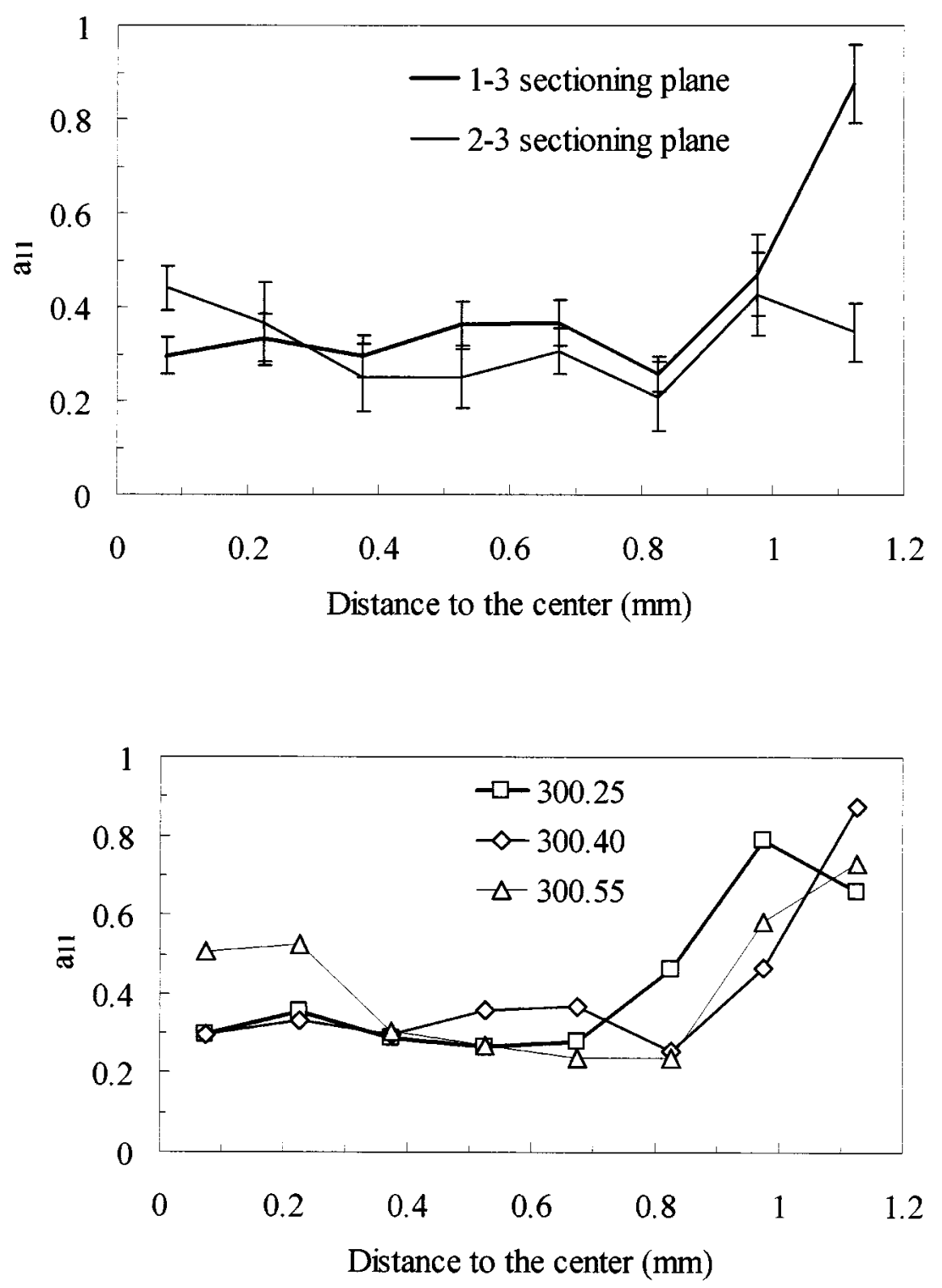

Fig. 12. Effect of injection speed in $a_{11}, 1-3$ sectioning plane, $300^{\circ} \mathrm{C}$ melt temperature.

Fig. 13. Effect of injection speed in $a_{11}, 1-3$ sectioning plane, $320^{\circ} \mathrm{C}$ melt-temperature.

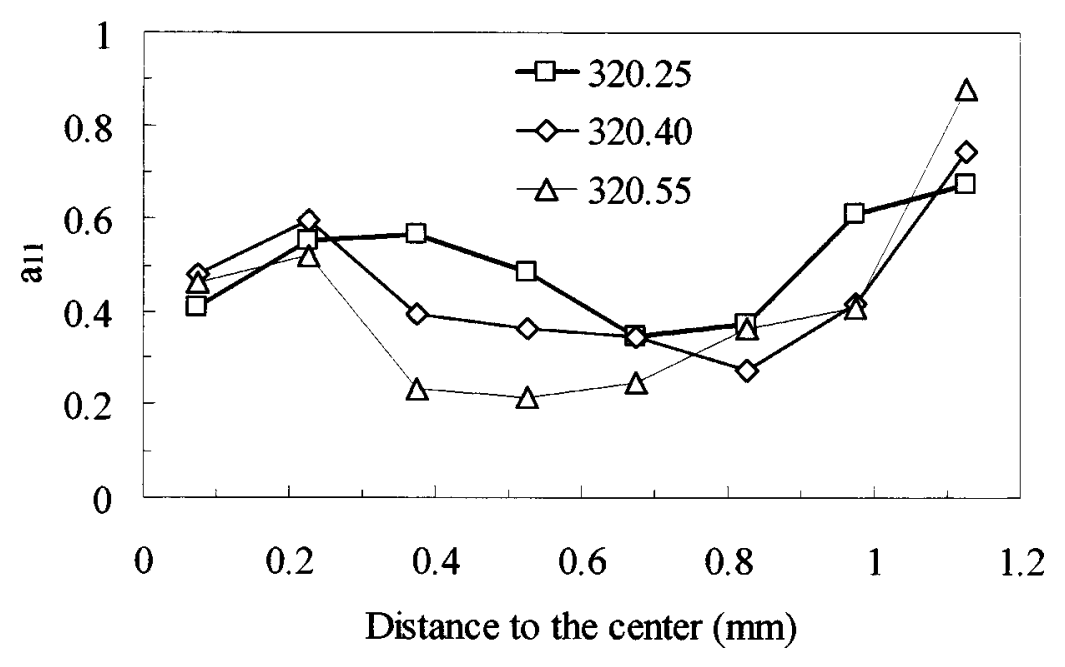

POLYMER COMPOSITES, OCTOBER 1998, Vol. 19, No. 5 


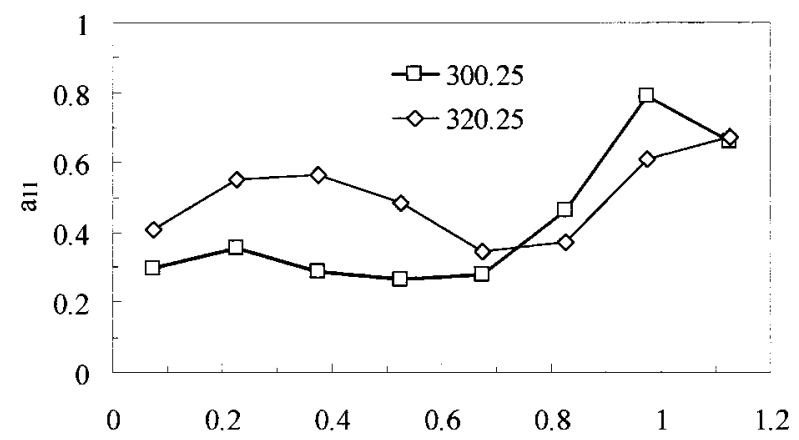

(a) Distance to the center (mm)

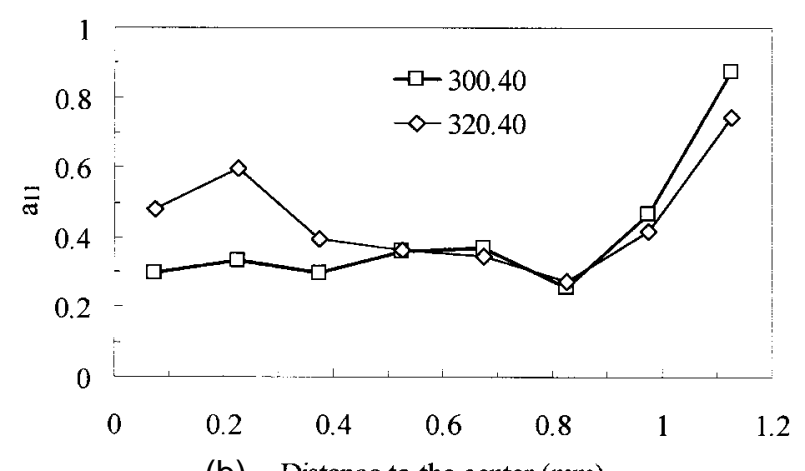

(b) Distance to the center (mm)

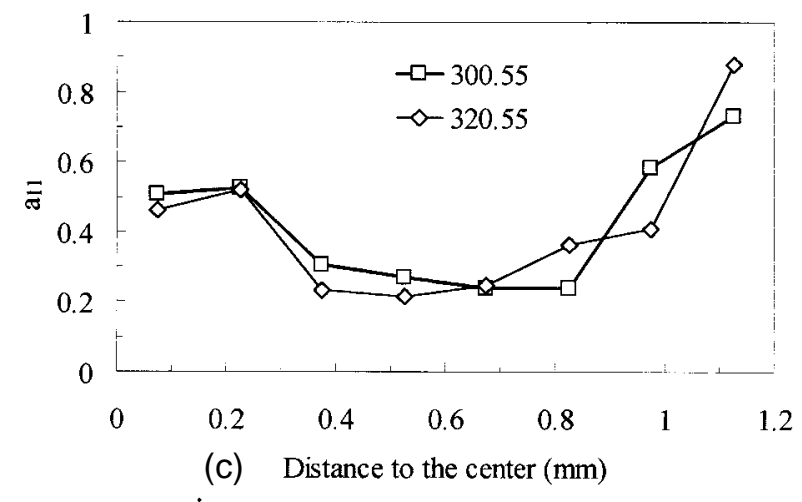

Fig. 14. Effect of melt-temperature in $a_{11}$, 1-3 sectioning plane. a) $25 \mathrm{~mm} / \mathrm{s}$, b) $40 \mathrm{~mm} / \mathrm{s}$ and c) $55 \mathrm{~mm} / \mathrm{s}$.

the core shows a higher fiber orientation transverse to the flow direction. Raising the melt temperature seems to favor a nearly random state of fiber orientation in the core $\left(a_{11} \approx a_{22} \approx 0.5\right)$. If higher injection speeds are used, the effect of the melt temperature is minimized (Fig. 14c).

Bay and Tucker (14) reported higher orientation levels along the flow direction of 4-mm-thick film gated plates, from PA 6.6 reinforced with long glass fibers. In their work, $a_{11}$ values of 0.8 in the skin and 0.2 in the core were observed, the thickness of the core being about $20 \%$ of the overall thickness. Similar high orientation results were also reported (15) using PA 6.6 reinforced with 43 wt $\%$ short glass fibers. Other authors $(21,32)$ report thick skin regions and high orientation level along the flow direction in heavily reinforced PC and PBT.

The $a_{11}$ values, measured in the core of the moldings in this work, are lower than those reported above. This may result from a less intense fiber interaction in the much less reinforced material used here (10 wt $\%$ vs. $40 \mathrm{wt} \%)$.

\subsection{Results From Bending Tests and Discussion}

The apparent moduli of elasticity in flexure experiments and those resulting from the FEM simulation using the orientation-averaged properties are summarized in Table 2 . It can be observed that the predictions are consistently higher than the experimental results. The average difference between simulations and experimental flexural stiffness is $8.3 \%$.

On increasing the injection speed a decrement of the apparent modulus in longitudinal direction will result. This is an expected result, since it was already shown that the fiber orientation in the flow direction is favored when the injection speed is lower. It can be observed that the across-flow direction specimens (label T) consistently present an apparent modulus higher than the corresponding along-flow direction specimens (label L). This fact contradicts intuitive expectations but it is clearly justified by the high transverse fiber orientation. Conversely, when the transverse specimens show lower stiffness, the longitudinal ones tend to exhibit higher stiffness, which demonstrates the complementary character of the $a_{11}$ and $a_{22}$ tensor components. A weak correlation between melt temperature and stiffness was observed.

The simulations are not in complete agreement with the experimental results. The simulations consistently overpredict the apparent modulus. The decreasing trend of the longitudinal flexural stiffness with increasing flow rate still can be observed in the simulations. However, the predicted flexural stiffness in the longitudinal direction is higher than in the transverse direction, thus contradicting the experimental results. The anisotropy ratio in the simulation decreases with increasing flow rate, which is in opposition to the trend observed in the experimental results.

The overprediction of the apparent modulus in the simulations may probably be related to the estimation of the properties using Halpin-Tsai equations. In fact in those equations, the stiffness very much depends on the aspect ratio of the fibers. The fiber length was experimentally determined, an average value was used for the calculations, but a very broad distribution was observed, with the standard deviation having values of the order of magnitude of the average.

\section{CONCLUSIONS}

One of the main objectives of this work was the characterization of fiber orientation in short fiber reinforced polycarbonate plates. It was intended to assess the predictions of mechanical properties based in fiber orientation measurements as well. 
Table 2. Experimental Flexural Stiffness and Apparent Flexural Stiffness Calculated From FEM Simulations. $L$ and $T$ Refer to Longitudinal and Transverse Specimen, Respectively.

\begin{tabular}{|c|c|c|c|}
\hline & $\begin{array}{c}\text { Experimental Flexural } \\
\text { Stiffness (Std. Dev.) } \\
\text { [MPa] }\end{array}$ & $\begin{array}{l}\text { Apparent Stiffness From } \\
\text { FEM Simulations } \\
\text { [MPa] }\end{array}$ & $\begin{array}{c}\text { Difference } \\
\text { [\%] }\end{array}$ \\
\hline 300.25.L & $2601.4(29.4)$ & 2974.5 & 12.5 \\
\hline 300.40.L & $2601.0(22.8)$ & 2973.0 & 12.5 \\
\hline 300.55.L & $2562.2(13.1)$ & 2886.3 & 11.2 \\
\hline $300.25 . T$ & 2634.7 (28.5) & 2818.4 & 6.5 \\
\hline 300.40.T & 2750.6 (13.2) & 2879.0 & 4.5 \\
\hline 300.55.T & $2720.2(23.0)$ & 2893.2 & 6.0 \\
\hline 320.25.L & $2691.0(30.2)$ & 2902.5 & 7.3 \\
\hline 320.40.L & $2634.4(40.5)$ & 2869.4 & 8.2 \\
\hline 320.55.L & $2532.0(16.0)$ & 2961.1 & 14.5 \\
\hline $320.25 . T$ & $2720.2(37.6)$ & 2820.9 & 3.6 \\
\hline $320.40 . T$ & 2744.9 (15.0) & 2909.4 & 5.7 \\
\hline 320.55.T & 2799.7 (10.7) & 3001.9 & 6.7 \\
\hline
\end{tabular}

The fiber orientation was observed to vary from the skin to the core. In the skin the fibers are always highly aligned in the flow direction, whereas in the core the fibers are mainly aligned in the direction transverse to the flow. However, the degree of transverse alignment and the thickness of the core were unexpectedly large.

The processing conditions affect the pattern of fiber orientation, the effect of injection speed being more pronounced than the melt temperature.

The experimental flexure tests showed that the stiffness in the flow direction decreases with increasing injection speed. The inverse was observed for transversely cut specimens. This is an expectable behavior, and was generally confirmed in the simulations. However, the experimental results indicate that the stiffness of the transverse specimens is higher than the longitudinal ones. This result was neither so expectable nor predicted in the simulations.

The orientation-averaging scheme allows the calculation of in-depth properties based in the fiber orientation measurements in spite of consistently overpredicting the stiffness of injection molded polycarbonate plates.

\section{ACKNOWLEDGMENTS}

N. M. Neves is supported by the Junta Nacional de Investigação Científica e Tecnológica, through a Portuguese based EU programme PRAXIS XXI grant, BD 3806/94.

The valuable and helpful suggestions of Dr. K. M. B. Jansen at The University of Twente are gratefully acknowledged.

The authors are indebted to Augusto Guimarães \& Irmão Lda, Valadares, Portugal, and to General Electric Plastics (Europe) at Bergen op Zoom, Holland, for supplying the materials used during this work.

\section{REFERENCES}

1. G. B. Jeffery, Proc. Roy. Soc., A102 (1922).

2. G. K. Batchelor, J. Fluid Mech., 41 (1970)
3. R. G. Cox, J. Fluid Mech., 44, 4 (1970).

4. G. K. Batchelor, J. Fluid Mech., 46, 4 (1971).

5. F. Folgar and C. L. Tucker III, J. Reinf. Plast. Compos., $\mathbf{3}$ (1984).

6. S. G. Advani and C. L. Tucker III, J. Rheology, 31, 8 (1987).

7. M. C. Altan, S. Subbiah, S. I. Güçeri, and R. B Pipes, Polym. Eng. Sci., 30, 14 (1990).

8. T. Matsuoka, J.-I. Takabatake, Y. Inoue, and H. Takahashi, Polym. Eng. Sci., 30, 16 (1990).

9. S. Akbar and M. C. Altan, Polym. Eng. Sci., 32, 12 (1992).

10. R. S. Bay and C. L. Tucker III, Polym. Compos., 13, 4 (1992).

11. S. Ranganathan and S. G. Advani, J. Non Newt. Fluid Mech., 47 (1993).

12. C. L. Tucker III and S. G. Advani, "Processing of ShortFiber Systems," in Flow and Rheology in Polymer Composites Manufacturing, S. G. Advani, ed., Comp. Mater. Series, Amsterdam (1994).

13. M. J. Crochet, F. Dupret, and V. Verleye, "Injection Molding," in Flow and Rheology in Polymer Composites Manufacturing, S. G. Advani, ed., Comp. Mater. Series, Amsterdam (1994).

14. R. S. Bay and C. L. Tucker III, Polym. Eng. Sci., 32, 4 (1992).

15. R. S. Bay and C. L. Tucker III, Polym. Compos., 13, 4 (1992).

16. S. Toll and P.-O. Anderson, Composites, 22, 4, (1991).

17. P. J. Hine, R. A. Ducket, N. Davidson, and A. R. Clarke, Compos. Sci. Technol., 47 (1993).

18. B. Möginger and P. Eyerer, Polymer, 22, 5 (1991).

19. A. Clarke, N. Davidson, and G. Archenhold, J. Microscopy, 171 (1993).

20. J. J. McGrath, B. Lian, and A. Nöthe, Proc. of ICCM-10, V-421 (1995).

21. B. Lian, A. Nothe, J. Ladewig, and J. J. McGrath, SPE ANTEC Tech. Papers, 41, 608 (1995).

22. M. W. Darlington and A. C. Smith, Polym. Compos., 8, 1 (1987).

23. K.-C. Ho and M.-C. Jeng, Plast. Rubb. Compos. Proc. Appl., 25, 10 (1996).

24. M. L. Dunn and H. Ledbetter, J. Acoust. Soc. Am., 99, 1 (1996).

25. S. Fakirov and C. Fakirova, Polym. Compos., 6, 41 (1985).

26. L. M. Gonzalez, F. L. Cumbrera, F. Sanchez-Bajo, and A. Pajares, Acta Metall. Mater., 42, 3 (1994).

27. M. McGee and R. L. McCullough, Polym. Eng. Sci., 34, 4 (1994). 
28. J. C. Halpin and J. L. Kardos, Polym. Eng. Sci., 16, 5 (1976).

29. J. E. Ashton, J. C. Halpin, and P. H. Petit, Primer in Composite Materials: Structure Property Relationships for Composite Materials, Technomic, Stamford, Conn. (1969).
30. S. G. Advani and C. L. Tucker III, SPE ANTEC Techn. Papers, 31, 1113 (1985).

31. M. Gupta and K. K. Wang, Polym. Compos., 14, 5 (1993).

32. R. M. Jones, Mechanics of Composite Materials, McGrawHill, Tokyo (1975). 